\title{
Highly coupled dielectric behavior of porous ceramics embedding a polymer
}

\author{
T. E. Gómez Alvarez-Arenas and F. Montero de Espinosa \\ Instituto de Acústica, Consejo Superior de Investigaciones Cientificas, C/Serrano 144, 28002, Madrid, \\ Spain
}

(Received 12 April 1995; accepted for publication 8 November 1995)

\begin{abstract}
We present a theoretical approach to study the dielectric properties of porous ceramics and composite materials and explain the experimentally observed notable influence of the coupling between the components of a saturated porous ceramic on the final behavior of the sample. This model is based on the assumption of a dielectric coupling between the components that modifies the expected averaged properties of the material. (C) 1996 American Institute of Physics.
\end{abstract}

[S0003-6951(96)00802-0]

In the last years, the heterostucture materials, such as composites of ceramics and polymers, ${ }^{1}$ porous ceramics, ${ }^{2,3}$ and nanocomposites ${ }^{4}$ have attracted much interest because of the enhanced properties that they exhibit in comparison to the single phase materials.

Usually, when quantum effects can be neglected, the electric, elastic, and piezoelectric properties of this type of materials are theoretically obtained by averaging approaches based on series and parallel models ${ }^{1}$ or on certain combinations of both. ${ }^{5,6}$ The global behavior of the composite is then obtained from the properties of each component and the structure. The first problem arises from the fact that the structure is not always known. In fact, in some cases, the differences between theoretical predictions and experimental results have been attributed to an error in the mathematical description of the structure. ${ }^{6}$ Furthermore, all of these models ignore any possible interactions between the components. In some cases, this interaction may be notable (as we report here) and should be considered in order to avoid errors in the interpretation of the observed behavior of such materials.

In the case of the elastic properties the theory developed by Biot $^{7}$ takes into account the mechanical interaction or coupling in fluid-solid composites, or even fluid-solid-gas composites. 8,9 This "coupling theory" introduces the new concept of poroelasticity. However, as far as we know, there is no coupling theory to study the dielectric properties in a composite.

The starting point of our approach is the derivation of the constitutive equations for the dielectric behavior of a composite following the same procedure as in Refs. 7 and 10 for the mechanical properties. If the dielectric losses can be neglected and only linear interactions are considered, then the Helmholtz free energy $(F)$ for a two components composite is given by the following quadratic form

$$
F=\frac{1}{2} P^{*} \chi_{n m} P_{n} P_{m}+\beta_{m} P_{n}^{*} P_{m}+\frac{1}{2}{ }^{P} \chi_{n m}^{*} P_{n}^{*} P_{m}^{*},
$$

where $P$ is the averaged polarization vector over a volume much higher than the typical composite substructuring, ${ }^{P} \chi$ is the inverse of the dielectric constant of each component at constant (or zero) polarization condition for the other one and $\beta$ is the coupling factor between them. The asterisk over $P$ and ${ }^{P} \chi$, as in other parameters, is used within the letter to distinguish one component from the other.

The exact differential of $F$ is given by $d F=E_{n} d P_{n}$ $+E_{n}^{*} d P_{n}^{*}$. Defining

$$
E_{n}=\left(\frac{\partial F}{\partial P_{n}}\right)_{P^{*}} \text { and } E_{n}^{*}=\left(\frac{\partial F}{\partial P_{n}^{*}}\right)_{P},
$$

it is easy to determine that the constitutive relation is composed of a set of coupled equations:

$E_{n}={ }^{P^{*}} \chi_{n m} P_{m}+\beta_{n m} P_{m}^{*} \quad$ and $\quad E_{n}^{*}={ }^{P} \chi_{n m}^{*} P_{m}^{*}+\beta_{n m} P_{m}$,

or the inverse relation

$P_{n}={ }^{*} k_{n m} E_{m}+\alpha_{n m} E_{m}^{*} \quad$ and $\quad P_{n}^{*}={ }^{E} k_{n m}^{*} E_{m}^{*}+\alpha_{n m} E_{m}$,

where $k=\epsilon-\epsilon_{0}, \epsilon$ is the dielectric permittivity, and $\epsilon_{0}$ is the dielectric permittivity of free space. The different dielectric parameters that appear in Eq. (4) are related in the following way:

$$
{ }^{P} k^{*}={ }^{E} k^{*}-\frac{\alpha^{2}}{E^{*} k} \quad \text { and } \quad P^{*} k={ }^{E^{*}} k-\frac{\alpha^{2}}{{ }^{E} k^{*}},
$$

where ${ }^{P} k\left({ }^{E} k\right)$ is the corresponding dielectric parameter of one component measured at constant polarization (electric field) conditions for the other one.

Considering $E=E^{*}$, as it can be done in many experimental cases then the measured dielectric permittivity of the composite is given by

$$
\boldsymbol{\epsilon}-\epsilon_{0}={ }^{E^{*}} k+{ }^{E} k^{*}+2 \alpha .
$$

The first step in validating this new model is to demonstrate that the influence of this coupling over the global behavior of the material cannot be justified or interpreted by other more classical methods, such as the averaging models (i.e., by changing the structure). We experimentally demonstrate that the variation of the dielectric permittivity of, for instance, a (3-3) composite when one of the components is changed without altering the structure, can only be justified in terms of the coupled theory presented here. 


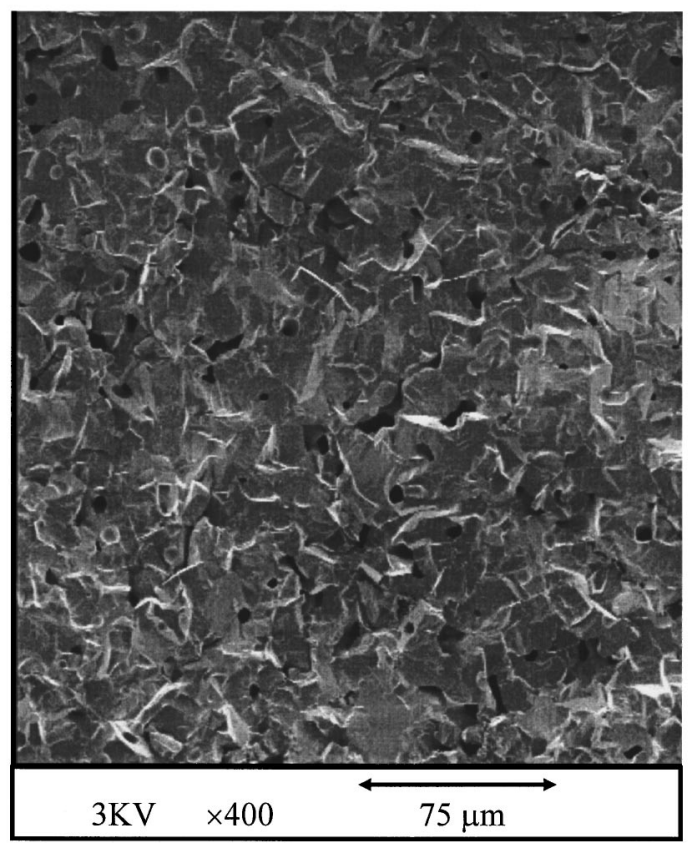

FIG. 1. SEM image of the porous ceramic.

For the experimental work it was important to choose a system in which the structure remained constant, but the properties of one of the phases could be altered. To this end we chose porous ceramics from a large set of commercial lead metaniobates. Only ceramics having the same dimensions, porosity $(\phi)$ and dielectric permittivity-when the interconnected pores are empty or air saturated $\left(\epsilon_{\text {empty }}\right)$ were chosen. The open porosity (in volume) was determined by weight measurements at empty pore and filled pore states obtaining a value of $(9.5 \pm 0.1) \%$ for the interconnected pores (otherwise the fluid cannot penetrate). This value, as well as the interconnected structure (3-3 connectivity) was verified by scanning electron microscopy (SEM) inspections of the sample as the micrograph of Fig. 1 reveals, where the size and structure of the pores can be clearly appreciated.

All the experiments followed the same scheme. First, we measured the dielectric permittivity of the porous ceramic with empty pores $\left(\epsilon_{\text {empty }}\right)$, from which we obtained the value of ${ }^{P} k^{*}=\epsilon_{\text {empty }}-(1-\phi) \epsilon_{0}$ (in this case, the asterisk denotes the properties of the ceramic). Then, we saturated the porous ceramic with a fluid by means of a vacuum technique. We verified the complete saturation of the interconnected pores by weight measurements with a precision of $10^{-4} \mathrm{~g}$ and a relative error in the measurement of about $10^{-2} \%$. A sample of the same fluid was placed between the plates of a specially designed condenser and we measured independently the dielectric permittivity of both the fluid saturated-porous ceramic $(\epsilon)$ and the bulk fluid $\left(\epsilon_{f}\right)$ with a $4194 \mathrm{~A}$ impedance analyzer (Hewlett-Packard).

The employed fluids were: a silicon oil and two epoxy resins: Eccogel 1365 and Araldite-H manufactured by Emerson and Cuming and Ciba and Geigy, respectively. The reason for using epoxy resins is that their dielectric properties change as a function of time during the curing [from 4 up to 8 for the relative dielectric permittivity $\left(\epsilon_{f} / \epsilon_{0}\right)$ while the

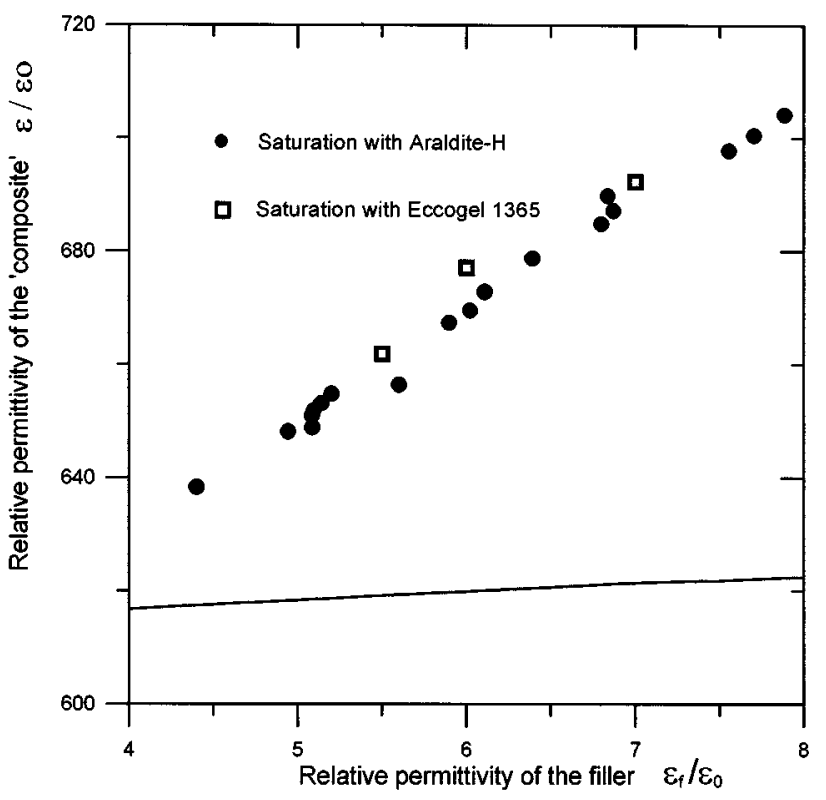

FIG. 2. Relative dielectric permittivity of the saturated porous ceramic vs the relative dielectric permittivity of the bulk filler $\left(\epsilon_{f} / \epsilon_{0}\right)$. Solid line: theoretical prediction of an averaging model (Banno) and experimental results for two different minor phases (fillers). (O): Araldite-H and ( $\square$ ): Eccogel 1365 .

dielectric losses are about $10^{-2}$ from gel point to solid state], it then being possible to obtain many different cases by monitoring the curing process. To this end, the dielectric permittivity of the epoxy-saturated porous ceramic $(\epsilon)$ and the (bulk) epoxy $\left(\epsilon_{f}\right)$ were measured over the same time period during the curing.

When the porous ceramic was saturated with silicon oil (relative dielectric permittivity $-\epsilon_{f}-$ of 3.2 ), the relative dielectric permittivity of the ceramic increased from $610 \pm 4$ $-\epsilon_{\text {empty }} / \epsilon_{0}-$ to $630 \pm 3-\epsilon / \epsilon_{0}-(3.3 \%)$. This increase cannot be explained by means of an averaging theory. For example, the relative increment expected from widely accepted modified cubes model of Banno ${ }^{11}$ is about $0.8 \%$.

A wider set of results is obtained when a porous ceramic is saturated with an epoxy, and the curing process is monitored as mentioned above. Figure 2 shows the relative dielectric permittivity of the saturated ceramic $(\epsilon)$ versus the relative dielectric permittivity of the minor phase filler $\left(\epsilon_{f}\right)$ for two different cases: saturation with Araldite-H and saturation with Eccogel. The different points in the graph correspond to different curing times in the epoxy evolution from gel to solid status. The figure also shows the predictions following Banno's approach. The incapacity of the averaging model (Ref. 11) to explain the notable increase of the ceramic dielectric permittivity when an epoxy resin is embedded in the pores can be appreciated.

From Eqs. (5) and (6) the variation of the dielectric permittivity from empty pore to filled pore conditions is given by:

$$
\epsilon-\epsilon_{\mathrm{empty}}=\Delta \epsilon={ }^{E^{*}} k+2 \alpha+\frac{\alpha^{2}}{E^{*} k} \text {. }
$$




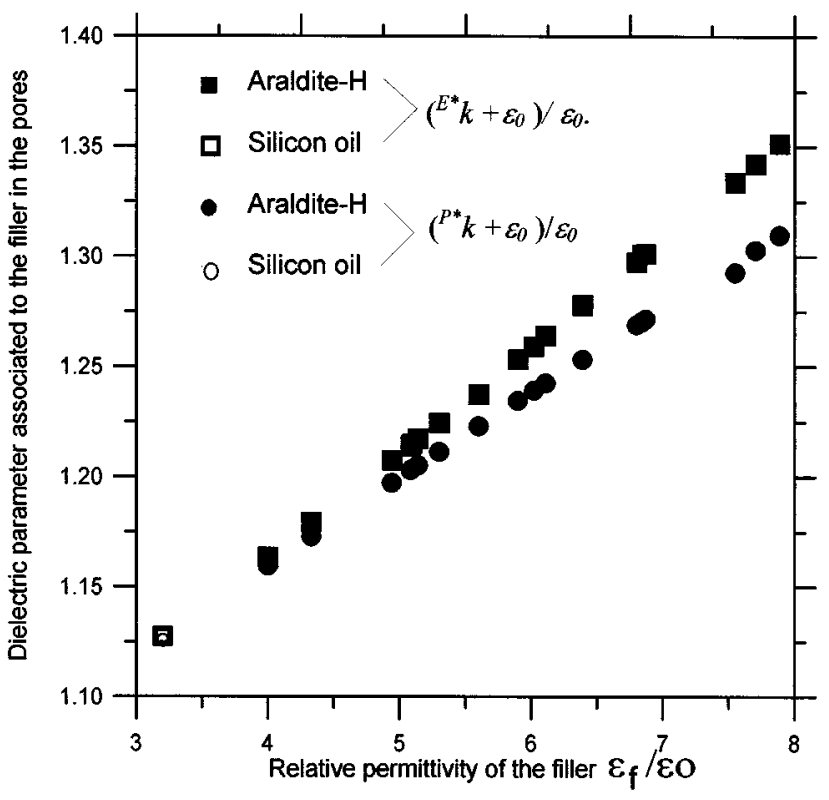

FIG. 3. Calculated dielectric parameters of the filler inside the pores $-\left({ }^{P^{*}} k+\epsilon_{0}\right) / \epsilon_{0}$ and $\left({ }^{E^{*}} k+\epsilon_{0}\right) / \epsilon_{0}-$ for fillers of different dielectric permittivity (following the curing for the epoxies) vs the measured relative dielectric permittivity of the bulk filler $\left(\epsilon_{f} / \epsilon_{0}\right)$.

Using the measured value of ${ }^{P} k^{*}$, the estimated value of $P^{*} k$ [obtained from Banno's model. In this case, the averaging model may be used because there is no dielectric coupling within a (3-3) structure in vacuum, the measured volume proportion of the minor phase $-\phi-$ and the measurements of $\boldsymbol{\epsilon}_{f}$ ] and the system of Eqs. (5) and (7), we obtain the magnitude of the dielectric coupling $(\alpha)$, as well as the value of the dielectric parameter that we have introduced: ${ }^{E} k^{*}$ and ${ }^{E^{*}} k$.

For saturation with silicon oil, and the procedure mentioned above, we obtained $\left({ }^{E} k^{*}+\epsilon_{0}\right) / \epsilon_{0}=1.1286,{ }^{E} k^{*} / \epsilon_{0}$ $=624$, and $\alpha / \epsilon_{0}=1.35$. These values fully explain the observed increase of the relative dielectric permittivity of the oil saturated ceramic.

Figure 3 shows the employed values of $\left({ }^{P^{*}} k+\epsilon_{0}\right) / \epsilon_{0}$ as well as the obtained values of $\left({ }^{E^{*}} k+\epsilon_{0}\right) / \epsilon_{0}$ [from Eqs. (5) and (7)] for all the experimental cases (silicon oil and epoxies). From the difference between these two quantities the dielectric coupling is calculated [Eq. (5)]. As expected, when the dielectric permittivity of the filling approaches one (vacuum), ${ }^{E *} k$ tends to equal ${ }^{P^{*}} k$ and, consequently, the dielectric coupling approaches zero $\left(\alpha^{2} \propto E^{*} k-P^{*} k\right)$. On the other hand, as the epoxy cures, ${ }^{E} k^{*} / \epsilon_{0}$ decreases from 695 to 628 and $\alpha / \epsilon_{0}$ also decreases from 5.5 to 1.5 . In addition, the permittivity of the oil-saturated ceramic (as well as ${ }^{E} k^{*} / \epsilon_{0}$ and $\alpha / \epsilon_{0}$ ) confirms the trend (with curing) of the measured permittivity of the polymer-saturated ceramics [as well as ${ }^{E} k^{*} / \epsilon_{0}$ (Fig. 3) and $\alpha / \epsilon_{0}$, respectively]. Therefore, we believe that the nature of this dielectric coupling is independent of the mechanical properties of the minor phase even for piezoelectric ceramics, although a more complete approach must be considered for this case (Ref. 12).

In summary, we have measured the increase of the dielectric constant of a porous ceramic when a second phase is embedded in the pores. We have shown that the measured values cannot be explained in terms of the averaging models with which this type of materials are usually studied. We have introduced a new approach that includes a dielectric coupling between the components. With this new approach we have calculated the coupling, explaining the measured extra increase of the dielectric constant.

The authors acknowledge financial support from the BRITE Contract BR2.CT94.1008. SEM photographs were performed by D. Martin of the CNM (CSIC) of Madrid.

${ }^{1}$ R. E. Newnham, D. P. Skinner, and L. E. Cros, Mater. Res. Bull. 13, 525 (1978).

${ }^{2}$ L. T. Canham, Appl. Phys. Lett. 57, 1046 (1990).

${ }^{3}$ T. Hayashi, S. Sugihara, and K. Okazaki, Jpn. J. Appl. Phys. 30, 2243 (1991).

${ }^{4}$ R. R. Oberle, M. R. Scanlon, R. C. Cammarata, and P. C. Searson, Appl. Phys. Lett. 66, 19 (1995).

${ }^{5}$ H. Banno, Jpn. J. Appl. Phys. 28, Suppl. 28-2, 190 (1989).

${ }^{6} \mathrm{H}$. Banno, Proceedings of 8th International Symposium on Applications of Ferroelectrics (IEEE-ISAF'94) P1-55 (1994).

${ }^{7}$ M. A. Biot, J. Acoust. Soc. Am. 28, 168 (1956).

${ }^{8}$ T. E. Gómez, E. Riera, and F. Montero, Ultrasonics 32, 131 (1994).

${ }^{9}$ T. E. Gómez, L. Elvira, and E. Riera, J. Appl. Phys. 78, 2843 (1995).

${ }^{10}$ M. A. Biot and D. G. Willis, J. Appl. Mech. 24, 594 (1957).

${ }^{11}$ H. Banno, Jpn. J. Appl. Phys. 32, 4214 (1993).

${ }^{12}$ T. E. Gómez and F. R. Montero de Espinosa (unpublished). 